\title{
Construcción sociocultural y vivencias COVID 19 del personal de atención primaria en salud
}

\author{
Sociocultural construction and COVID 19 experiences of primary health care personnel
}

\section{Construção sociocultural e experiências COVID 19 de pessoal de atenção primária à saúde}

\author{
Matilde Nohemí Dávila-Otero \\ mndavilao@utn.edu.ec \\ https://orcid.org/0000-0002-9834-1246
}

\author{
Widmark Enrique Báez-Morales \\ webaez@utn.edu.ec \\ https://orcid.org/0000-0003-3996-0925
}

\author{
Mercedes del Carmen Flores-Grijalva \\ mcflores@utn.edu.ec \\ https://orcid.org/0000-0001-5582-4507 \\ Universidad Técnica del Norte, Ibarra-Ecuador
}

Recibido 24 de febrero 2021 | Arbitrado y aceptado 2 de marzo 2021 | Publicado en 4 de mayo 2021

\begin{abstract}
RESUMEN
Los estudios socioculturales constituyen un campo del saber interdisciplinario que han generado nuevas teorías y procesos renovados de elaboración social de las ciencias de la salud. Objetivos. En el contexto de la COVID-19, permitieron conocer, comprender e interpretar los fundamentos, significados y vivencias del personal de primer nivel de atención en salud. Método. Se empleó investigación biográfico-narrativa, como técnicas se usó las entrevistas, análisis de contenido y triangulación, los instrumentos que se utilizaron: fueron la ficha de registro documental, batería de preguntas y matrices de análisis de categorías deductivas. Resultados. Para la presentación de los resultados y su posterior discusión, se usaron figuras tales como: nubes de palabras, tablas de resumen y redes semánticas diseñadas con el apoyo del programa ATLAS.ti; se citaron, además, múltiples comentarios expresados por los entrevistados; y, se triangularon con base en las fuentes, teorías y métodos consultados en bases académicas de alto impacto. Conclusión. A final, se concluyó que más allá de la fundamentación teórico-metodológica imprescindible y que requiere de actualizaciones incesantes, es necesario valorar al ser humano invisibilizado tras un perfil profesional y laboral; se encontraron, asimismo, doce significados socioculturales construidos por el personal de salud: apatía, desorientación distanciamiento, inmutabilidad, integralidad, miedo, pandemia, perfeccionamiento, religiosidad, resignación, salud y trabajo en equipo. Como palabras generadoras de las vivencias narradas, se hallaron ocho: catarsis, familia, humanidad, incertidumbre, virtualidad, vulnerabilidad, espiritualidad y conflicto existencial.
\end{abstract}

Palabras clave: Construcción sociocultural; COVID-19; personal de salud; vivencias

\begin{abstract}
Sociocultural studies constitute an interdisciplinary field of knowledge that has generated new theories and renewed processes of social elaboration of health sciences. Objectives. In the context of COVID-19, they made it possible to know, understand and interpret the foundations, meanings and experiences of first level health care personnel. Method Biographical-narrative research was employed, using interviews, content analysis and triangulation as techniques. The instruments used were the documentary record card, the battery of questions and the analysis matrices of deductive categories. Results. For the presentation of the results and their subsequent discussion, figures such as word clouds, summary tables and semantic networks designed with the support of the ATLAS.ti program were used; multiple comments expressed by the interviewees were also cited; and they were triangulated based on the sources, theories and methods consulted in high-impact academic databases. Conclusion. In the end, it was concluded that beyond the essential theoretical-methodological foundation that requires constant updating, it is necessary to value the human being invisible behind a professional and labor profile; twelve socio-cultural meanings constructed by the health personnel were also found: apathy, disorientation, distancing, immutability, integrality, fear, pandemic, improvement, religiosity, resignation, health and teamwork. Eight words were found to generate the experiences narrated: catharsis, family, humanity, uncertainty, virtuality, vulnerability, spirituality and existential conflict.
\end{abstract} MD: Licenciada en enfermería, maestranda en
Salud Pública, mención enfermería familiar y comunitaria. Enfermera del Centro de Salud №1. Ibarra-Ecuador. Universidad Técnica del Norte, Imbabura Ecuador.

WB: Magíster en Gerencia en Salud para el desarrollo local de la Universidad Técnica desarricular de Loja - Ecuador. Doctor en Medicina y Cirugía de la Universidad Central del Ecuador. Profesor auxiliar, Universidad Técnica del Norte - Ibarra-Ecuador, en la Facultad de Ciencias de la Salud, y profesor de llamada en la Maestría de Salud Pública de la misma universidad. Universidad Técnica del Norte, Imbabura Ecuador. MF: Magister en Salud Pública, Mención Investigación en Sistemas y Servicios de Salud. Universidad Técnicara de Norte Coordinadora la Maestría Salud Pública, Mención Enfermería la Maestria Salud Pública, Mención Enfermería Familiar y Comunitaria del Instituto de
Posgrado de la Universidad Técnica del Norte, Imbabura Ecuador. 

MD: Licenciada en enfermería, maestranda en comunitaria. Enfermera del Centro de Salud №1. Ibarra-Ecuador. Universidad Técnica del Norte, Imbabura Ecuador

WB: Magister en Gerencia en Salud para el desarrollo local de la Universidad Técnica Particular de Loja - Ecuador. Doctor en Medicina yirugía de la Universidad Central de Ecuador. Profesor auxilar, Universidad Técnica del Norte - lbarra-Ecuador, en la Facultad de Maestría de Salud Prública de llamada en la universidad. Universidad Técnica del Norte Imbabura Ecuador.

MF: Magister en Salud Pública, Mención Investigación en Sistemas y Servicios de Salud. Docente en la Carrera de Enfermería de la Universidad Técnica del Norte. Coordinadora de la Maestría Salud Pública, Mención Enfermería Familiar y Comunitaria del Instituto de Posgrado de la Universidad Técnica del Norte

\begin{abstract}
RESUMO
Os estudos socioculturais constituem um campo interdisciplinar de conhecimento que tem gerado novas teorias e processos renovados de elaboração social das ciências da saúde. Objetivos. No contexto da COVID-19, eles permitiram conhecer, compreender e interpretar os fundamentos, significados e experiências do pessoal de saúde de primeiro nível. Método. Foi utilizada a pesquisa biográfico-narrativa, como técnicas foram utilizadas as entrevistas, análise de conteúdo e triangulação, os instrumentos utilizados foram o cartão de registro documental, bateria de perguntas e matrizes de análise de categorias dedutivas. Resultados. Para a apresentação dos resultados e sua posterior discussão, foram utilizadas figuras como: nuvens de palavras, tabelas de resumo e redes semânticas projetadas com o apoio do programa ATLAS.ti; múltiplos comentários expressos pelos entrevistados também foram citados; e, foram triangulados com base nas fontes, teorias e métodos consultados em bases acadêmicas de alto impacto. Conclusão. No final, concluiuse que, além dos fundamentos teórico-metodológicos essenciais que exigem atualizações incessantes, é necessário valorizar o ser humano invisível atrás de um perfil profissional e trabalhista; doze significados socioculturais construídos pelo pessoal de saúde também foram encontrados: apatia, desorientação, distanciamento, imutabilidade, integralidade, medo, pandemia, aperfeiçoamento, religiosidade, resignação, saúde e trabalho em equipe. Como palavras geradoras das experiências narradas, foram encontradas oito: catarse, família, humanidade, incerteza, virtualidade, vulnerabilidade, espiritualidade e conflito existencial.
\end{abstract}

Palavras-chave: Construção sociocultural; COVID-19; pessoal de saúde; experiências; profissionais de saúde

\section{INTRODUCCIÓN}

$\mathrm{L}$ os estudios socioculturales (1), se centran en la interrelación entre individuo, sociedad, comunicación y cultura. Si bien se expresa que se gestaron a partir de 1960, para explicar los complejos fenómenos socioculturales producidos después de la segunda guerra mundial; Daniels (2), destaca que fue James Wertsch quien utilizó originalmente el término «sociocultural» para establecer la forma de funcionamiento mental de los seres humanos y su interrelación en los diversos contextos sociales, culturales e históricos. Se reconoce, además, como antecedente al término "sociohistórico" como legado de Lev Vygotsky.

En Latinoamérica, el filósofo ecuatoriano Bolívar Echeverría (3), integra a la cultura como una dimensión fundamental de la vida social. Esta interpretación, que parece actual, tiene antecedentes en los estudios de Edward Tylor, fundador de la antropología social, quién en 1871, ya había definido la cultura como una construcción social (4):

La cultura [...] en su sentido
etnográfico amplio, es ese
complejo universo que
incluye el conocimiento, las
creencias, el arte, la moral, la
ley, las costumbres, así como
otras capacidades y hábitos
adquiridos por el hombre
como miembro de una
sociedad.

Sobre la base de los planteamientos y referentes teóricos apuntados, se define la construcción sociocultural como la integración e interdependencia de lo social y lo cultural desde una visión de elaboración y mediación social de la realidad.

Por otra parte, en el contexto de la pandemia COVID-19, la ciudadanía en general y el personal de salud en particular, han buscado transmutar las comunicaciones cotidianas que muestran una visión 
meramente estadística de la tragedia. Más allá de las cifras de afectados y muertos, existen vivencias de seres humanos que cohabitan y luchan cotidianamente contra este flagelo.

En consonancia con la función biopsicosocial atribuida al personal de atención primaria en salud, sería inconsecuente perder de vista las vivencias y los significados socioculturales construidos por enfermeras, médicos, psicólogos, odontólogos, obstetras, auxiliares de farmacia, laboratoristas y personal administrativo. Son ellos, quienes diariamente perciben situaciones de dolor, lucha, incertidumbre, desesperación, vulnerabilidad e impotencia: verdaderas historias de vida que se pierden dentro de las cifras actualizadas y publicadas cotidianamente por la prensa, radio, televisión y redes sociales (5).

De cara a esta realidad, resulta pertinente y responsable, describir y narrar vivencias que muestren también otras facetas del profesional calificado temporalmente como héroe. Con ello, se espera que la sociedad comprenda la dimensión humana del profesional sanitario, con sus fortalezas, debilidades, ideas, comportamientos y emociones.

La COVID-19, representa un padecimiento que ocasionó una pandemia que tiene y tendrá enormes repercusiones para la humanidad, en los ámbitos socioeconómicos, culturales, políticos... pero más que nada, en la cotidianidad de los seres humanos $(6,7)$. Esta enfermedad ha dejado huellas indelebles en la sociedad contemporánea. De manera particular, en el personal de salud que enfrenta cada día, una situación de salud pública para la cual no estuvo preparado. Ante esta enfermedad, el equipo de primer nivel de atención en salud, también ha experimentado mayor presión, escasez de recursos, impotencia y temores ante la falta de estudios que estandaricen adecuadas maneras de enfrentarla.

En este contexto especificado, se planteó: conocer, comprender e interpretar la construcción sociocultural producto de las vivencias del personal sanitario que atiende a la variedad de pacientes que tienen esta patología, en el marco de su trabajo profesional.

Con relación al evento de estudio, se encontró que, en el mundo, se han efectuado múltiples investigaciones desde varios ámbitos del quehacer académico y científico. "Disease surveillance: how might surveillance studies address COVID-19?" (5), es posiblemente uno de los pocos estudios contemporáneos que relacionan la construcción sociocultural con la COVID-19. A pesar de que se enmarca en la vigilancia epidemiológica, sus hallazgos son reveladores cuando aportan tres construcciones socioculturales: (in) seguridad, vulnerabilidad y riesgo.

En otro estudio (8): "Experiencias de vida y soporte percibido por las enfermeras de las unidades de hemodiálisis hospitalaria durante la pandemia de COVID-19 en España", se concluye que coexisten emociones positivas y negativas provocadas por el desconocimiento de protocolos de actuación y miedo al contagio, agravado por la falta de medios de protección. Como vivencias resaltan: el crecimiento personal, el apoyo de los colegas y de la familia. No obstante, la investigación se ajusta exclusivamente al personal de enfermería de atención hospitalaria en un contexto sociocultural europeo. 
Como parte de la revisión sistemática (9): "Impacto de la epidemia del Coronavirus (COVID-19) en la salud mental del personal de salud y en la población general de China", se determinó como factores de impacto psicológico en enfermeras, médicos $\mathrm{y}$ estudiantes de Wuhan, localidad de posible origen de la pandemia: estrés, ansiedad y depresión, asociados al sexo femenino, a la condición de estudiantes, a síntomas físicos específicos y a una pobre percepción de la propia salud. Al final, como desafío, se sugirió el cuidado de la salud mental del personal de salud y la población en general.

Referenciales pero distantes de las vivencias del personal de salud, son dos estudios realizados en ámbitos educativos $(10,11):$ "Prevalence and socio-demographic correlates of psychological health problems in Chinese adolescents during the outbreak of COVID-19" y "Learning and Teaching Online During COVID-19: Experiences of Student Teachers in an Early Childhood Education Practicum". En el primero, se establece problemas de estrés, ansiedad y depresión en estudiantes chinos de 12 a 18 años; $y$, en el segundo, hallazgos positivos y negativos del proceso enseñanzaaprendizaje en línea.

Entre las investigaciones sobre vivencias, resaltan: "The power of story: narrative inquiry as a methodology in nursing research" (12); y, "La vivencia y su análisis: consideraciones breves sobre las nociones objeto-sujeto en el universo discursivo del mundo cultural" (13). Estas referencias, más allá de aportar evidencias relacionables con el evento de estudio, dan pautas de la metodología biográficonarrativa en estudios con enfermeras y desde punto de vista de los estudios culturales.

\section{MATERIALES Y MÉTODOS}

$\mathrm{S}$ e trató de una investigación biográficanarrativa, vinculada a los planteamientos de la construcción sociocultural. Como técnicas se utilizó: revisión documental, entrevistas, análisis de contenido, con el apoyo del programa ATLAS.ti y triangulación con base en fuentes, teorías y métodos. Entre los instrumentos se utilizaron: fichas de registro documental, batería de preguntas y matrices de análisis de categorías deductivas. La unidad de estudio estuvo integrada por ocho trabajadores de la salud: médico, enfermera, obstetra, odontólogo, psicóloga, laboratorista, auxiliar de enfermería y auxiliar administrativo de salud, que laboran en el Centro de Salud $N^{\circ} 1$ de Ibarra-Ecuador. El criterio de inclusión fue de informantes críticos, como voceros de los departamentos profesionales establecidos en la Unidad de Salud. Se excluyeron aquellos trabajadores que se encuentran en teletrabajo. En atención a criterios bioéticos, cada participante suscribió un consentimiento informado, luego de las explicaciones detalladas sobre el objetivo investigativo, el tratamiento confidencial de la información y la posibilidad de abandonar el estudio en el momento que decidieran.

Para ello, fueron desarrollados tres categorías y códigos planteados en la investigación relacionados con los fundamentos, significados y vivencias, la cual se muestra detalladamente en el apartado de los resultados y discusión, seguidamente representados en las figuras y tablas; se citan, además, algunos comentarios expresados por los entrevistados; y luego, se triangulan con los hallazgos encontrados y priorizados de artículos científicos y libros 
divulgados en bases de datos como: PubMed, Scielo, Scopus, Springer, Taylor \& Francis y Web of Science.

A continuación, en la Tabla 1, se detalla el proceso de determinación de las "categorías deductivas" que orientaron el proceso de recolección y análisis de datos. Con su empleo, se construyeron las fichas de registro documental de artículos científicos y libros que sirvieron de base para la fundamentación teórica y la triangulación necesaria en la interpretación de los hallazgos, como también para la elaboración de la guía de entrevista conformada por 18 preguntas, que fue aplicada al personal de atención primaria en salud.

Tabla 1. Categorías deductivas sobre construcción sociocultural y vivencias COVID-19.

\begin{tabular}{ll}
\hline \multicolumn{1}{c}{$\begin{array}{c}\text { Categoría o } \\
\text { concepto central }\end{array}$} & \multicolumn{1}{c}{ Definición } \\
\hline Fundamentos & $\begin{array}{l}\text { Teoría y metodología que } \\
\text { teórico- }\end{array}$ \\
sustentan la percepción y \\
construcción & $\begin{array}{l}\text { comprensión de la realidad de } \\
\text { parte de una persona o } \\
\text { sociocultural y }\end{array}$ \\
Sociedad con sustento en los \\
mecanismos de construcción \\
social y cultural en el contexto \\
de la COVID-19.
\end{tabular}

\begin{abstract}
Significados socioculturales interiorizados por el personal de atención primaria en salud.
\end{abstract}

Eventos biográficos (Vivencias) del personal del Centro de Salud $N^{\circ} 1$ en el contexto COVID-19.
Sistema de ideas o representaciones sociales $\mathrm{y}$ culturales del personal de atención primaria en salud con sustento en sus saberes profesionales, saberes basados en la experiencia y teorías implícitas.

Hechos, sentimientos, actitudes y vivencias narradas por sí mismo u otra persona en los cuales se mencionan logros y fracasos en la trayectoria vital del personal de atención primaria de salud en el contexto de la Covid 19.

\section{Códigos}

Construcción social de la realidad (sentido, representaciones e ideologías)

Construcción cultural de la realidad (dimensión cultural, mito arte y ciencia, comunicación y mediación) COVID-19 (agente etiológico, epidemiología, manifestaciones clínicas diagnóstico, tratamiento y vacunas)
Saberes profesionales (sentido y representaciones de conocimiento personal y profesional)
Saberes basados en la experiencia (praxis profesional e intereses de la política sanitaria)
Teorías implícitas (fundamentos teóricos de referencia y normas de convivencia)

Personal de salud (Planificación de actividades cotidianas, rituales profesionales, comprensión y toma de decisiones)

Vivencias Covid 19 (anécdotas personales y profesionales, expresiones reiteradas, creencias, valores, rasgos y tradiciones, preocupaciones, necesidades de información y capacitación y ejemplos de vida) 


\section{RESULTADOS Y DISCUSIÓN}

\section{Fundamentos teórico-metodológicos sobre construcción sociocultural y COVID-19}

Se delinearon dos bases teóricas la primera relacionada con la construcción sociocultural, desglosada en dos temas (construcción social de la realidad y la construcción cultural de la realidad); y, vivencias COVID-19 del personal de salud, en tres (COVID-19, personal de salud y vivencias). Para el análisis y discusión, se aplicó los códigos expuestos en la Tabla 1, para elaborar las tablas y figuras. En la figura 1, se expone una nube de palabras que ilustra aquellas expresadas con mayor frecuencia; y, en la tabla 2, se presenta una síntesis de la información analizada en la red construida.

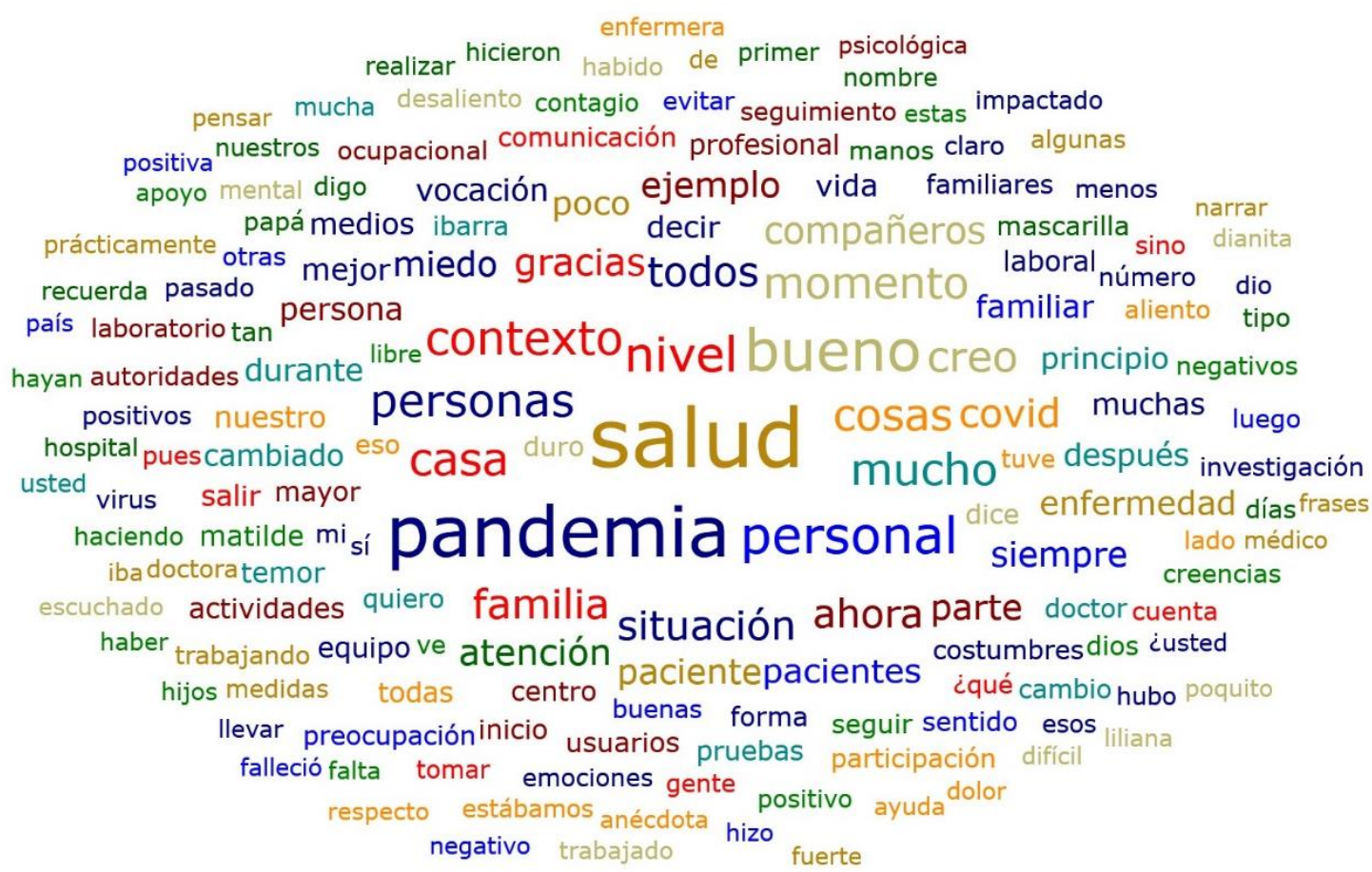

Figura 1. Nube de palabras: fundamentos sobre construcción sociocultural y vivencias COVID-19.

Entre los conceptos que adquirieron mayor relevancia para el personal de primer nivel de atención en salud, se priorizan, con base en la alta frecuencia expresada, así como derivación de la triangulación realizada: salud, pandemia, personal, contexto, familia, paciente $\mathrm{y}$ COVID-19. Seguidamente se apuntan algunos comentarios expresados por los consultados y que se relacionan con fundamentos teórico-metodológicos.

- Claro, bueno, si hemos tenido compañeros que han tenido pérdidas cercanas de su círculo familiar, y eso si ha sido super, super duro ver ese sufrimiento de cerca, eso entonces, ver ese sufrimiento cercano, esa 
realidad, si, si fue super duro. Al inicio pues, uno después va analizando y dice "bueno y si le hubieran dado esta medicación, si se hubiera manejado así y eso", hace un recuento, pero ya pues, la perdida está y no hay nada que hacer. (Obstetra).

- Al principio, como era una enfermedad nueva, pues y fue difícil de afrontarla, pues con el miedo, con los cuidados que, en ese momento, pues estaban recientes, o sea no había no había métodos ni un proceso, digamos, establecido para el protocolo, digamos establecido para el manejo de enfermedades. Entonces en ese momento lo afrontamos, tal vez al principio con el criterio primero de los cuidados y segundo el distanciamiento, alejamiento, porque conocíamos muy poco sobre la enfermedad. (Odontólogo).

- Me acuerdo del artista que falleció y como que era algo público y pensamos que nada más y ya, porque él venía supuestamente de España y que allá se contagió y ya, pero, después ya tocaba hacer los seguimientos a los pacientes y era el miedo, no, porque era una enfermedad nueva, no se tenía el tratamiento hasta ahora, pues no tenemos un tratamiento para esa enfermedad y entonces empezaron las muertes. Entonces todo ha sido terrible, terrible perder compañeros a nivel nacional de otros países y también familiares. Eso ha tocado mucho el corazón de todos, como profesionales y también como personas. (Médico familiar).

- Si hay creencias que dicen que tienen ya el remedio. No sé si puedo hablar como creencias o que ya encontraron la cura. Porque si he escuchado ya tratamientos basados en medicina natural, "eh", ocupan bastante lo que es trago puntas que se lo llama. También he escuchado que utilizan una planta que se llama cascarilla, "eh". Como cura que comen ajo que comen bastante ajo, en dosis no. En dosificación en la mañana y en la tarde he escuchado que se comen eso. Utilizan medicamentos que no están autorizados por ninguna entidad de salud como es la Ivermectina sea inyectable u oral. Entonces, "eh", no sé si lo puedo llamar como creencias, pero ya hay parte de la sociedad que ya tiene aparentemente entre comillas un tratamiento, pero que no está validado por ninguna entidad de salud nacional e internacional. Entonces cada quien llama el grupo social que maneja a su manera ya lo que es la situación del COVID. Entonces ya tienen sus curas y sus y sus formas de cuidarse, por lo que el señor dice yo sé cómo me cuido, yo me cuido con eso. Yo utilizo lo otro y yo me como este otro. Entonces ya dicen yo con eso me curo. (Enfermera).

- Y pues sí, sí, sigue siendo a veces como que mueve las emociones de una porque recuerda muchas experiencias, especialmente de personas, familias, y cómo le decía pacientes no, que tuve la oportunidad de hacer muchos acompañamientos, incluso a compañeros de salud, que qué si conmovía ¿no?, entonces incluso uno como psicólogos también yo corrí donde mis compañeros para también tener esa fortaleza para poder fortalecer a mis compañeros y a todas las personas que que necesitaban en ese momento y que siguen necesitando, entonces fue esa, ese 
también saber que que uno como psicólogo también se quiebra y que debe buscar también ese ese apoyo, esa pirámide, esa fortaleza ¿no?, de de otro tipo de, de la formación personal que uno tiene. Entonces todo eso me ayudó y me sigue ayudando a fortalecerme todos los días para poder seguir apoyando a las personas que que todavía sufren mucho por el tema del COVID y por los estragos y por la situación de salud mental que hoy en día se ve muchísimo, muchísimo. (Psicóloga).

- Con lágrimas en los ojos yo me fui a mi primera toma de muestras para COVID y recuerdo que al llegar a la casa de paciente en donde iba a tomar la primera muestra, me recibió la familia del paciente, me trasladaron a su lugar donde estaba aislado el paciente y el paciente que estaba sentado en su cama me dice, señorita, antes de que me tome la muestra yo quiero saber si voy a vivir o voy a morir, ¿usted me puede decir eso antes de hacerme el examen? Entonces yo no sabía que contestarle, que decirle en ese momento en el que yo también me encontraba con miedo, tal vez con más miedo que él y lo único que le supe decir era que nosotros íbamos a tratar de ayudar y que íbamos a poner todo de nuestra parte para poder ayudar y sanarle y a lo que me dijo, señorita, yo desde hoy le voy a rezar al dios que está en el cielo para que le ayude y le guie en todo su trabajo para que ni a usted ni a sus compañeros les pase nada. (Laboratorista).

- Que a veces no hay mucho material y a veces carecemos de fundas, carecemos de desinfectantes, que a veces no hay cloro o que no hay esto. Entonces tenemos que esperar hasta cuando venga la otra remesa de materiales que hagan el pedido en la zona para que lleguen y nos puedan dar y si no toca hacer milagros. Y que importante es que nosotros limpiemos bien, aunque sólo con agüita, pero limpiemos bien. Eso es lo importante. (Auxiliar administrativa de salud).

- O sea, lo que más me sentí, me dio, o sea, como le digo, sentimiento, me dio tristeza de la paciente, más que por mí, o sea, de la paciente, porque era una paciente de la tercera edad, y yo, o sea, usualmente soy muy sentimental y entonces sentí mucho dolor, como se dice, como si fuese un familiar mío. (Auxiliar de farmacia).

Tabla 2. Expresiones reiteradas por el personal de atención primaria.

\begin{tabular}{|c|c|c|}
\hline Categorías & Códigos & $\begin{array}{c}\text { Expresiones del personal de atención } \\
\text { primaria }\end{array}$ \\
\hline $\begin{array}{l}\text { Fundamentos } \\
\text { teórico- } \\
\text { metodológicos sobre } \\
\text { construcción } \\
\text { sociocultural y } \\
\text { COVID-19 }\end{array}$ & $\begin{array}{l}\text { Construcción social de la } \\
\text { realidad (sentido, } \\
\text { representaciones e } \\
\text { ideologías) }\end{array}$ & $\begin{array}{l}\text { Miedo de contagiarse y contagiar a la familia, } \\
\text { desacato de las disposiciones de parte de la } \\
\text { población, bajón anímico de los profesionales } \\
\text { ubicados en primera línea, sentimientos de } \\
\text { vulnerabilidad y riesgo continuo, realizar } \\
\text { protocolos de desinfección al ingresar al } \\
\text { hogar para evitar contagios de la familia. }\end{array}$ \\
\hline
\end{tabular}


Tabla 2. Expresiones reiteradas por el personal de atención primaria.

\begin{tabular}{|c|c|c|}
\hline Categorías & Códigos & $\begin{array}{c}\text { Expresiones del personal de atención } \\
\text { primaria }\end{array}$ \\
\hline $\begin{array}{c}\text { Fundamentos } \\
\text { teórico- } \\
\text { metodológicos sobre }\end{array}$ & $\begin{array}{l}\text { Construcción cultural de } \\
\text { la realidad (dimensión } \\
\text { cultural, mito arte y } \\
\text { ciencia, comunicación y } \\
\text { mediación) }\end{array}$ & $\begin{array}{l}\text { Confinamiento fue positivo para la naturaleza } \\
\text { (disminución contaminación), pasar más } \\
\text { tiempo con la familia, limitada importancia a } \\
\text { la enfermedad de parte de la población, } \\
\text { desinfección permanente de ropa, calzado y } \\
\text { superficies de contacto, requerimiento } \\
\text { permanente de apoyo a las familias con } \\
\text { parientes contagiados, persistencia de } \\
\text { reuniones sociales y familiares, existe mayor } \\
\text { disciplina en otros países. }\end{array}$ \\
\hline $\begin{array}{l}\text { construcción } \\
\text { sociocultural y } \\
\text { COVID-19 }\end{array}$ & $\begin{array}{l}\text { COVID-19 (agente } \\
\text { etiológico, epidemiología, } \\
\text { manifestaciones clínicas } \\
\text { diagnóstico, tratamiento y } \\
\text { vacunas) }\end{array}$ & $\begin{array}{l}\text { Confinamiento por la pandemia, ideas } \\
\text { erradas de parte de la población con relación } \\
\text { a la enfermedad, percepción poblacional de } \\
\text { que la pandemia era y es una mentira, } \\
\text { población se cuida con medicamentos } \\
\text { caseros, inadecuado uso de mascarilla de } \\
\text { parte de la población, seguir normas de } \\
\text { bioseguridad, tranquilidad a partir de la } \\
\text { llegada de las vacunas, mayor cantidad de } \\
\text { contagios en las reuniones familiares. }\end{array}$ \\
\hline
\end{tabular}

Como parte de esta investigación se encontró creencias y acciones reiteradas que se implementan en el contexto de la pandemia, a saber: ideas erradas y de negación de la pandemia de parte de la población que motivan el desacato permanente de las normativas impuestas por las autoridades nacionales, regionales y locales. Automedicación e incumplimiento del distanciamiento físico y de las normas de bioseguridad, lo que ha conducido a un incremento sobredimensionado de los casos de COVID-19 y fallecimiento de un alto número de pacientes. Frente a ello, el personal de salud incrementó, hasta niveles considerados de pánico (7), las normas de asepsia y bioseguridad, como el lavado permanente de manos, el uso de doble mascarilla y respiradores N95 y KN95, prendas de protección personal y pantallas de protección facial.
Estos aspectos relatados: miedo a contagiar a su familia, tratamientos $\mathrm{y}$ sustancias no acreditadas por la comunidad científica mundial y desorientación teóricometodológica, a pesar de la formación profesional adquirida, no se han considerado en los estudios científicos divulgados $\mathrm{y}$ disponibles en las bases de datos antes expuestas.

Con base en el análisis y discusión realizada con esta categoría de estudio, se logró determinar cuatro constructos socioculturales: desorientación, miedo, salud y pandemia.

\section{Significados socioculturales interiorizados por el personal de atención primaria en salud}

En este acápite se muestran los hallazgos relacionados con los significados socioculturales que ha interiorizado el personal de primer nivel de atención en 
salud. En la figura 2, se ilustra una red que muestra las relaciones establecidas; $\mathrm{y}$, en la Tabla 3, una síntesis de los principales significados construidos.

Debido a la importancia y enfoque desarrollado en este estudio, en primer lugar, para esta categoría, se citan varias de las expresiones narradas por los entrevistados:

- Ahora como que ya hemos perdido el efecto de héroes como era al principio, eso también decepciona, porque bueno en principio que nos alababan todo, que nos admiraban y así, pero ahora es como que ya, pues, no están haciendo nada fuera de lo común, ósea no importa si se contagian o no importa o sea ya no importa, ya, ya pasó y seguimos en lo mismo, porque ahora se está repitiendo la historia, con esa diferencia de que ya no hay esa consideración al personal de salud. (Obstetra).

- Si vamos por el lado de las autoridades, en el sitio de la enfermedad, pues las autoridades nos negaran el uso de protección. Pensaban que estábamos llegando con un miedo a la gente a mostrarnos nosotros muy protegidos y las demás personas que todavía por las calles sin protecciones. (Odontólogo).

- A veces es como que uno se siente. Desalentadas, digamos, por las propias autoridades. Al principio nos decían que por qué nos ponemos la doble mascarilla y que no sé cuánto, porque hacemos tal o cual cosa y así ¿no?, entonces en eso sí nos ha desalentado. Entonces, hasta ahora usted ve, no se ha cumplido la ley humanitaria, por ejemplo. Entonces esas cosas de parte de las autoridades desalientan. (Médico familiar).

- Pues pese a las precauciones que se tuvieron en un momento salí COVID positiva, si eso me afectó bastante psicológica y emocionalmente. $\mathrm{Y}$ dando gracias a Dios fue leve, sintomatología leve, nos recuperamos la familia y todo, pero me dejó con una sensación aún más mayor de temor frente a la situación, no ya que me contagié de la manera menos esperada. En mi preocupación a nivel personal. Dejar a mis hijos sin madre, no a mí. (Enfermera).

- Si yo creería que sí, sí, sí Se dio ese aporte de todos. Ya el equipo completo que ya el laboratorio, que ya psicología, que ya enfermería, que ya medicina, entonces si se consolida día a día ese equipo y en el centro de salud, por lo menos yo así lo miro y a mí siempre me me han incluido en esa situación y siempre me me dicen tengo tal paciente, ayúdame, entonces yo si miro que hay ese esa inclusión en salud mental que en años anteriores probablemente tal vez no se hubiera visto, ¿no? (Psicóloga).

- Creo que las creencias religiosas, muy aparte de la religión que se tenga, esas también se fortalecieron mucho, yo creo que todo ser humano cree en un ser superior que nos guía, yo creo que, en esta pandemia, con todo lo que se vivió, se fortaleció en todos los servidores de la salud. (Laboratorista).

- Bueno, parece que aquí les ha faltado un poquito de organización, pero ahorita ya creo que están organizados. Sí. Lamentablemente en esto de las vacunas y todo eso estaba un poquito desorganizado, pero ya, ya veo que la 
cogieron por el ritmo. Todo anda bien, porque yo también me he ido, me he ido igual con los doctores así ayudándoles. Pero ya está todo bien, para mí está todo bien. O sea, lo que mis ojos ven ahora no se... (Auxiliar administrativa de salud).

- Las preocupaciones que hemos tenido es que, o sea que he tenido yo personalmente es que me vaya a contagiar y vaya a contagiar a otras personas. (Auxiliar de farmacia).

Sobre la base del análisis de los comentarios y la red semántica ilustrada en la figura 2, se determinaron, seis constructos socioculturales: 1. apatía, 2. distanciamiento, 3. inmutabilidad, 4 . religiosidad, 5. resignación, 6. trabajo en equipo. El primero $\mathrm{y}$ el tercero, reconocidos en las acciones de las autoridades y muchos usuarios de los servicios de salud; los restantes, priorizados en las narraciones del personal de salud.

Con relación a la aparente apatía e inmutabilidad determinada y de exclusiva responsabilidad de algunas autoridades, varios sectores de la población y parte del personal de salud, Paolo Giordano (6), escribió:

No tengo miedo de caer enfermo. ¿Y de qué tengo miedo? De todo lo que el contagio puede cambiar. De descubrir que el andamiaje de la civilización que conozco es un castillo de naipes. De que todo se derrumbe, pero también de lo contrario: de que el miedo pase en vano, sin dejar ningún cambio tras de sí.

Con una idea parecida Slavoj Žižek (7), citando a Hegel, expresa: [...] lo único que podemos aprender de la historia es que no aprendemos nada de la historia, así que dudo que la epidemia nos haga más sabios. Sin ubicarse en los extremos de desconfianza e incredulidad en las posibilidades para el aprendizaje vivencial de los seres humanos, los resultados encontrados parecen confirmar las apreciaciones de los dos pensadores referidos. 


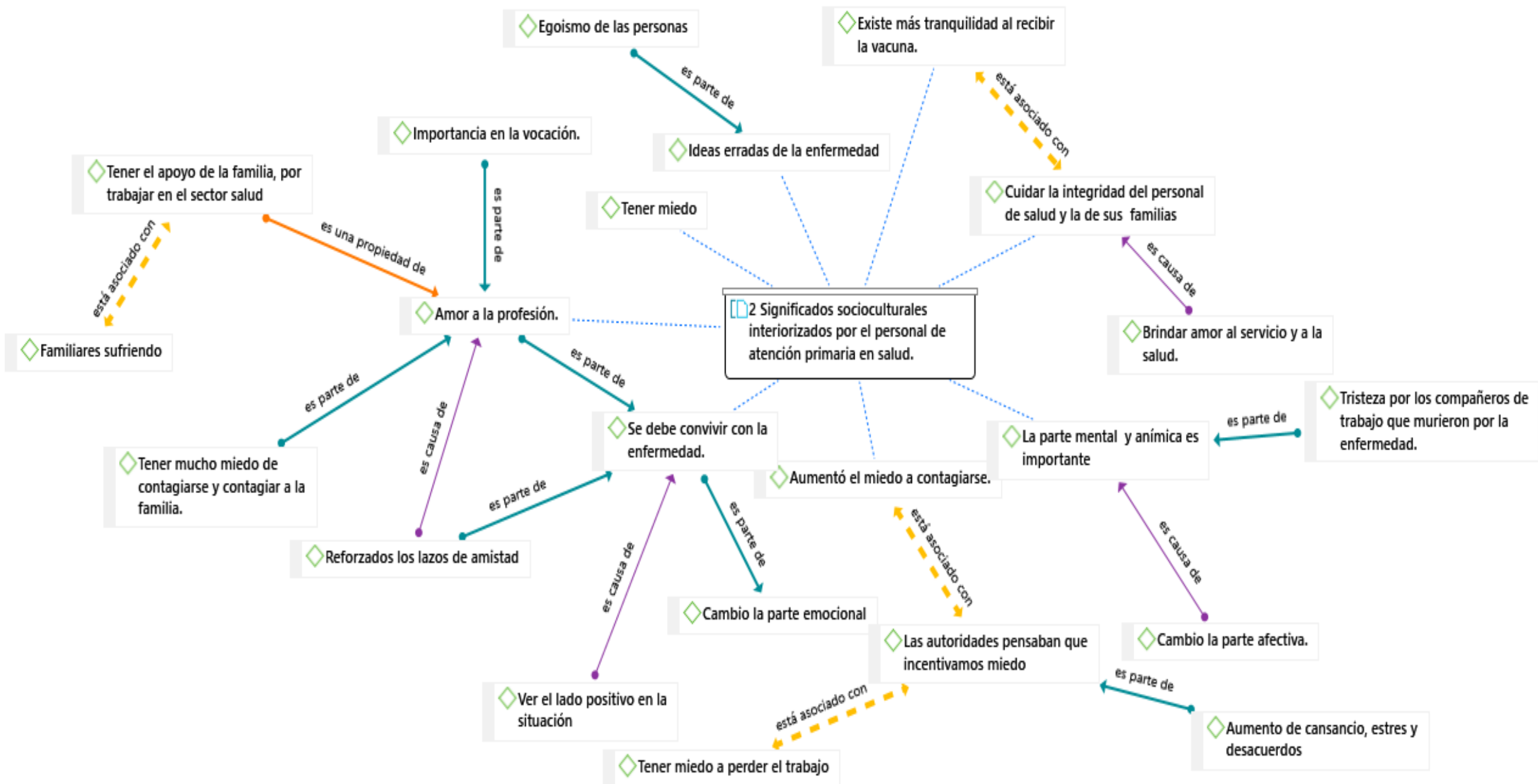

Figura 2. Red de significados socioculturales interiorizados por el personal de salud. 
Parte de los hallazgos relatados encontraron sustento en al menos dos de los estudios realizados en el contexto de la pandemia $(8,9)$; así como en documentos sobre estudios culturales (14) y análisis de datos cualitativos (15). En ellos, también se evidencian relaciones con al menos cinco de los significados construidos: apatía, integralidad, miedo, trabajo en equipo y resignación.

Tabla 3. Expresiones reiteradas por el personal de atención primaria.

\begin{tabular}{|c|c|c|}
\hline Categorías & Códigos & $\begin{array}{c}\text { Expresiones del personal de atención } \\
\text { primaria }\end{array}$ \\
\hline \multirow{3}{*}{$\begin{array}{l}\text { Significados } \\
\text { socioculturales } \\
\text { interiorizados por el } \\
\text { personal de } \\
\text { atención primaria } \\
\text { en salud. }\end{array}$} & $\begin{array}{l}\text { Saberes profesionales } \\
\text { (sentido y } \\
\text { representaciones de } \\
\text { conocimiento personal y } \\
\text { profesional) }\end{array}$ & $\begin{array}{l}\text { Mayor cuidado en el sitio de trabajo, escasa } \\
\text { información y capacitación del personal de } \\
\text { salud, estrés y tristeza por la muerte de } \\
\text { compañeros trabajadores de la salud, } \\
\text { resurgimiento de la vocación por la profesión. }\end{array}$ \\
\hline & $\begin{array}{l}\text { Saberes basados en la } \\
\text { experiencia (praxis } \\
\text { profesional e intereses de } \\
\text { la política sanitaria) }\end{array}$ & $\begin{array}{l}\text { Las actividades de la unidad de atención móvil } \\
\text { eran correctas, continuar con las } \\
\text { responsabilidades en el trabajo, la } \\
\text { implementación de políticas sanitarias ha sido } \\
\text { débil, necesidad de cambios de las políticas de } \\
\text { dotación de insumos. }\end{array}$ \\
\hline & $\begin{array}{l}\text { Teorías implícitas } \\
\text { (fundamentos teóricos de } \\
\text { referencia y normas de } \\
\text { convivencia) }\end{array}$ & $\begin{array}{l}\text { Realizar aislamiento de la familia, incremento } \\
\text { de la empatía entre compañeros, egoísmo de } \\
\text { parte de la población y débil empatía } \\
\text { comunitaria, apoyo profesional en las visitas } \\
\text { domiciliarias, aprender a convivir con la } \\
\text { enfermedad, las autoridades no dan normas } \\
\text { claras que se basen en la realidad del } \\
\text { contexto, necesidades de perfeccionamiento y } \\
\text { formación en salud. }\end{array}$ \\
\hline
\end{tabular}

Los principales hallazgos ilustrados en la Tabla 3, dan cuenta de la desactualización de las políticas sanitarias, sobre todo con relación a la dotación de insumos, protocolos de acción y actividades de aprendizaje y formación que, para el personal de salud, han sido limitados durante casi toda la vigencia de la pandemia.
Por otra parte, a más de los seis constructos antes evidenciados, luego del análisis y discusión a partir de las entrevistas y la red semántica de la Figura 2; con la revisión de la Tabla 3, se suman dos nuevos constructos, estos son: integralidad y perfeccionamiento. 


\section{Eventos biográficos (Vivencias) del personal del Centro de Salud $\mathrm{N}^{\circ} 1$ en el contexto COVID-19}

Por último, se muestran las rutinas exteriorizadas de parte del personal de salud. Para ello, en la Figura 3, se ilustra en una red semántica sobre eventos biográficos; ocho citas extraídas de las entrevistas que narran algunas vivencias significativas; $\mathrm{y}$, en la tabla 4 , varias expresiones reiteradas de parte del personal de salud.

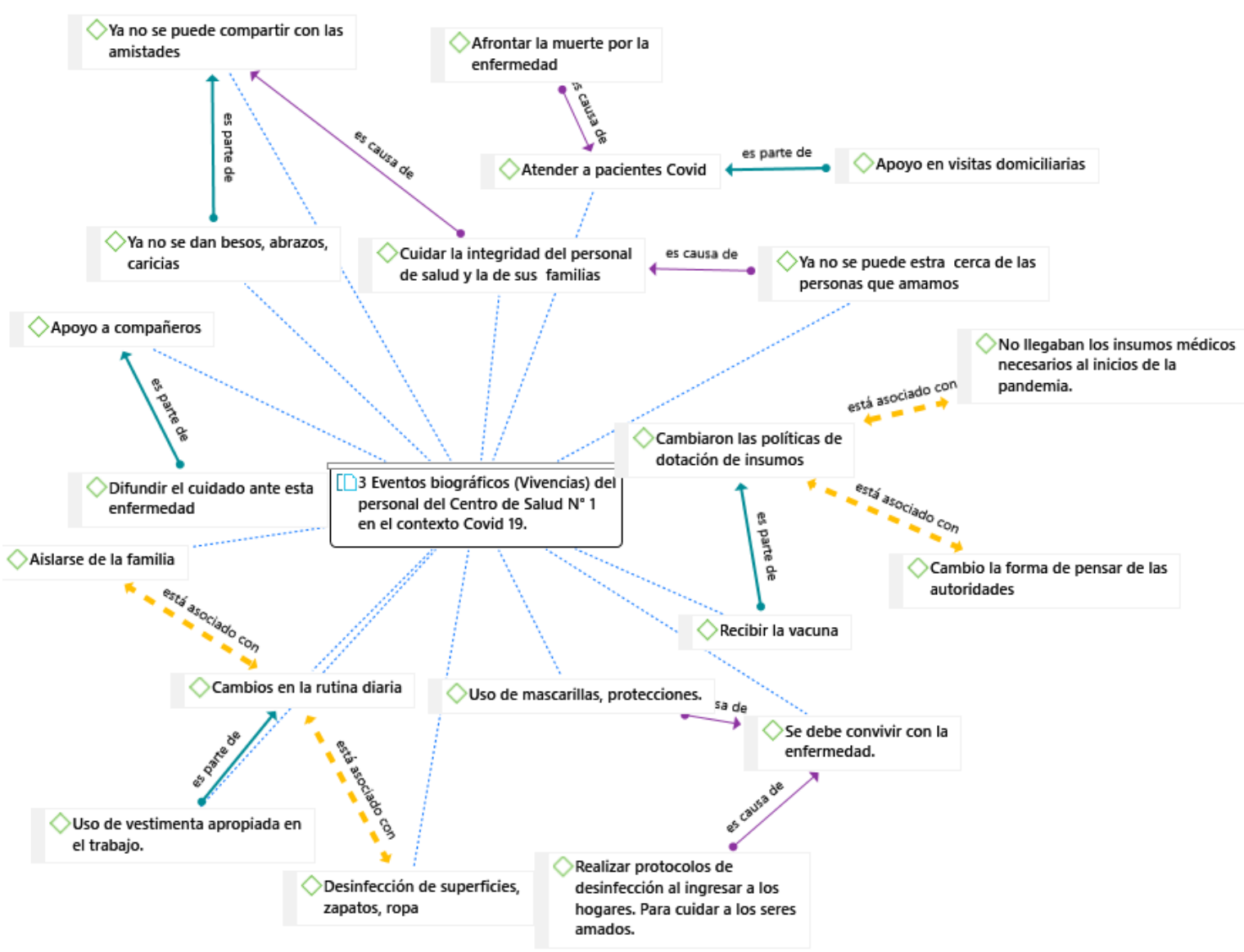

Figura 3. Vivencias del personal de atención primaria en salud.

Entre las vivencias más significativas destacan aquellas referidas a los abrazos y besos virtuales, la vestimenta que confunde al personal de salud con personajes de ficción y la cercanía de la muerte en casi todos los contextos de la interacción humana. En varias de las anécdotas narradas por el personal de primer nivel de atención en salud, se puede corroborar lo manifestado:
- Bueno, la anécdota sería que hemos atendido pacientes que han sido positivos y que ellos no sabían tampoco que eran positivos o a lo mejor lo sospechaban, pero a veces uno quiere coger y decir, se niega al principio, no, no, no, no es, no es esto no es, esto es diferente de lo que dicen, no los síntomas, porque a cada 
quien le coge diferente. Y ya pues, después con el susto de que han sido positivas y como ya dije antes, uno se pone a pensar a ver que le hice, estuve muy cerca, le topé y así, entonces hace una recapitulación de todo lo que hizo con esa persona, eso sería. (Obstetra).

- Bueno, hubieron varios casos, en las visitas, pues puede ser, por ejemplo acudí a una emergencia donde la madre de la casa, digamos así, la cabeza de hogar, pues se enfermó y pues tuvo que ser llevada en la noche en ambulancia porque ya se puso en estado crítico. Entonces eso fue muy duro para las hijas y para nosotros como personal de salud que acudían en ese momento de emergencia, ver desvanecerse casi una vida y pues, luego la señora nos enteramos que murió, entonces fue muy difícil afrontar eso, la muerte por enfermedad, que es lo más grave. (Odontólogo).

- Bueno, pues contarle que, en mi familia tuvimos dos pérdidas, ¿no? Primero, al principio de la pandemia, ya prácticamente este 11 de abril es un año, de que falleció mi papi. Como les decía al principio, él estuvo trabajando en contra de él y de toda la familia. Él estaba trabajando por disposición de su jefe. Como digo, todo era nuevo. ¿no? Entonces empezó con fiebre, con fiebre. En la casa estuvo aislado. Yo tuve una noche que irle a atender y por eso a mí también me aislaron, que prácticamente ahora vemos que no era necesario. Pero en ese tiempo teníamos miedo, digamos, y no sabíamos qué hacer. Pero bueno, en ese momento me aislaron también y prácticamente yo hacía videollamada con mi papá. Viendo como está, le hacía el seguimiento un médico de aquí del centro de salud, le hacía el seguimiento en la casa y después de eso se agravó. Estuvo unos días en el hospital del IESS hasta que ya hubo la necesidad de intubarlo, paso unos cuantos días intubado y al final falleció. Entonces eso ha sido muy duro, porque primero como hija, como profesional de salud. Perder a un familiar, no sólo a un familiar, a mi padre, sí ha sido muy, muy duro. A pesar de que ya ha pasado un año, pero todavía es muy, muy fuerte. Para mí es muy, muy reciente el dolor que se siente de la pérdida, pues, nosotros éramos muy unidos. Siempre las cosas hacíamos juntos. Mi papá nos dio siempre el ejemplo del trabajo, de la responsabilidad $\mathrm{y}$ eso es lo que nosotros nos llevamos. Él hasta el último estuvo cumpliendo su trabajo y pues así, así falleció. Tal vez se pudo hacer algo más. Tal vez, no sé. Pero esos son los designios de Dios y uno no puede ir contra eso. Y ya. Dios creo que ya se lo llevó porque ya era su momento. Pero para los que quedamos aquí. Pues sí, es muy, muy duro que él ya no esté, porque era nuestra, nuestra cabeza, nuestro ejemplo. Como profesional de salud sí me tocó mucho, porque cada vez que atendía un paciente revivía esos momentos con mi papi. Tenía miedo de que lo que me estaba pasando a mí le vuelva a pasar a otro, a otra persona. (Médico familiar).

- Recuerdo que una persona adulta mayor asistió a la unidad. La paciente llegó en busca de ayuda, atención médica. Se sentía mal y al controlarle signos vitales estaban inestables desaturaba. Su frecuencia cardíaca 
estaba elevada y mencionaba sensación de ahogo, de falta de aire y jadeaba mucho. Le molestaba la mascarilla. Y tengo un conocimiento que minutos después fue cuestión de minutos, falleció. Entonces eso fue muy estremecedor e impactante personalmente. (Enfermera).

- Bueno, de desaliento a veces este tema de la inconciencia, ¿no?, que uno está todos los días ahí, dale y dale, luchando para ver la mejora en los pacientes, sí que existe mucha inconciencia de las personas alrededor, ¿no?, salen, no usan bien la mascarilla, no hay distanciamiento, entonces, ese cómo, como reprocha la ciudadanía que uno escucha y que uno también a veces como que se tiene que morder la lengua para no decir "ay, Dios mío". (Psicóloga).

- Hubo un momento de inestabilidad laboral dentro de nuestro trabajo que también nos puso a pensar que para que nos esforzábamos tanto a cumplir nuestro trabajo, que nuestros superiores no lo sabían valorar, ahí solo éramos como una prenda descartable que la usaron y que se podía desechar sin importar en nuestros hombros llevábamos a una familia y que habíamos arriesgado el bienestar de esa familia por estar dando todo por nuestro trabajo. (Laboratorista).

- Sí, sí, sí. Ha habido un cambio. No porque queremos hacerlo, pero sí ha habido cambios. Sí, sí, a veces tal vez yo también me siento mal, o tal vez le haga sentir mal a otra persona, pero a veces nos alejamos un poquito. 0 tal vez no puedo tomar con todos el café. No, que mínimo debe haber aquí unas dos personas, lo normal... Entonces van unos más allá otros más acá. Conversamos asimismo de lejitos y eso sí, si ha habido cambios, incluso a veces en la forma de saludar. Solo que de repente nos olvidamos y claro nos damos un abrazo. Pero bueno, como estamos protegidos...digo bueno. (Auxiliar administrativa de salud).

- A ver, una experiencia que tuvimos aquí en el centro de salud fue verá que, a ver, vino una paciente y que falleció en procedimientos, ahí tuve, o sea, como le digo, o sea un miedo, ¿por qué? Porque yo estuve ahí, fui allá y dijeron este paciente está con COVID, o sea, me sentí mal porque dije o sea ya me contagié. Tuve miedo ese momento. (Auxiliar de farmacia). 
Tabla 4. Expresiones reiteradas por el personal de atención primaria.

\begin{tabular}{|c|c|c|}
\hline Categorías & Códigos & $\begin{array}{c}\text { Expresiones del personal de atención } \\
\text { primaria }\end{array}$ \\
\hline & $\begin{array}{l}\text { Personal de salud } \\
\text { (Planificación de } \\
\text { actividades cotidianas, } \\
\text { rituales profesionales, } \\
\text { comprensión y toma de } \\
\text { decisiones) }\end{array}$ & $\begin{array}{l}\text { Temor de que no paguen los sueldos, apoyo } \\
\text { del departamento de salud ocupacional, } \\
\text { insuficiente organización de parte de } \\
\text { autoridades, las autoridades pensaban que } \\
\text { los profesionales motivaban el miedo en la } \\
\text { población, uso de mascarillas y equipo de } \\
\text { protección como un ritual cotidiano, } \\
\text { difusión permanente de normas de } \\
\text { bioseguridad y distanciamiento físico. }\end{array}$ \\
\hline $\begin{array}{l}\text { Eventos biográficos } \\
\text { (Vivencias) del } \\
\text { personal del Centro } \\
\text { de Salud } N^{\circ} 1 \text { en el } \\
\text { contexto COVID- } 19 .\end{array}$ & $\begin{array}{c}\text { Vivencias Covid } 19 \\
\text { (anécdotas personales y } \\
\text { profesionales, } \\
\text { expresiones reiteradas, } \\
\text { creencias, valores, rasgos } \\
\text { y tradiciones, } \\
\text { preocupaciones, } \\
\text { necesidades de } \\
\text { información y } \\
\text { capacitación y ejemplos } \\
\text { de vida) }\end{array}$ & $\begin{array}{l}\text { Dolor por el fallecimiento de pacientes con } \\
\text { COVID-19, gratitud de pacientes } \\
\text { recuperados de COVID, no llegan a tiempo } \\
\text { insumos necesarios para tratamiento, } \\
\text { escaso reconocimiento al personal de salud } \\
\text { del primer nivel, miedo a perder el trabajo, } \\
\text { disminución de celebraciones, incremento } \\
\text { del cansancio, estrés y desacuerdos, } \\
\text { limitaciones de la afectividad y compartir } \\
\text { con compañeros y amistades (menos } \\
\text { abrazos y muestras de afecto), afrontar la } \\
\text { muerte cotidiana de conocidos y } \\
\text { desconocidos, cambios en la rutina diaria, } \\
\text { incremento de las dificultades de } \\
\text { comunicación con los pacientes por el uso } \\
\text { de mascarillas y protecciones. }\end{array}$ \\
\hline
\end{tabular}

A más de lo referido, una de las vivencias que han quitado el sueño, a no pocos profesionales de la salud, es la relacionada con la posibilidad de perder su trabajo debido a la mala situación socioeconómica que el país vive como consecuencia del quiebre de muchas empresas y el limitado ingreso de recursos a las arcas fiscales del Estado. Esta situación, de acuerdo con varias noticias divulgadas por algunos medios de comunicación, ha determinado que al menos un millón de ecuatorianos hayan perdido su trabajo y cinco millones convivan en situaciones laborales precarias (16).

De otro lado, sobresalen las percepciones de escritores (6), filósofos (7), autoridades y población en general, con relación a las muestras de un exagerado temor y hasta el pánico por el cumplimiento de normas de bioseguridad y el distanciamiento físico, que en ocasiones ha devenido en un distanciamiento social.

Este constructo: distanciamiento social, ha sido cuestionado por Bradley Hilgert, PhD. (17), y otros ponentes del webinar: Impactos socioculturales del COVID-19 en Guayaquil, Ecuador: La intensificación de desigualdades. Para el académico referido, ese constructo vulnera la necesidad de una convivencia armónica entre los distintos pueblos y representa, además, una relación intransigente de construcción sociocultural. 
A pesar de las connotaciones negativas, el constructo distanciamiento, adjetivado como social, se ha divulgado tanto que se usa cotidianamente en los múltiples escenarios de vida, así como a través de los medios de comunicación y redes sociales. De ahí que se haya convertido en un estereotipo que también encasilla y vulnera los derechos sociales de quienes padecen COVID-19.

Por último, como vivencias reiteradas se narran aquellas relacionadas con la virtualidad de las relaciones laborales, profesionales y hasta familiares; la vulnerabilidad profesional y social; la incertidumbre sobre el presente y futuro de la humanidad; la valoración espiritual y menoscabo de lo material; y, el conflicto existencial vida-muerte.

\section{CONCLUSIONES}

$\mathrm{L}$ a COVID-19 y sus múltiples construcciones socioculturales, constituye un nuevo escenario de fundamentación teórico-metodológica que se debe abordar desde la comunidad científica y académica, los medios de comunicación y las instituciones gubernamentales y no gubernamentales. Más allá de la demostración experimental, las cifras sin rostro comunicadas día a día, así como el énfasis en los procesos administrativos institucionales; es necesaria también, la valoración de los significados y vivencias desde el punto de vista de los seres humanos que están tras de un perfil profesional y laboral.

Como producto de la participación de los representantes de cada departamento que conforman la institución de salud estudiada, se encontraron doce significados socioculturales construidos por el personal de salud, a saber: apatía, desorientación, distanciamiento, inmutabilidad, integralidad, miedo, pandemia, perfeccionamiento, religiosidad, resignación, salud y trabajo en equipo.

Asociadas a las vivencias narradas por el personal de primer nivel de atención en salud, se encontró ocho palabras generadoras: catarsis, familia, humanidad, incertidumbre, virtualidad, vulnerabilidad, espiritualidad y conflicto existencial. Al final, su uso reiterado en los discursos narrados por los entrevistados, posibilitaron obtener una valoración de cómo estas, han influido en su interpretación vivencial en los ámbitos personal y profesional.

\section{REFERENCIAS BIBLIOGRÁFICAS}

1. O'Sullivan T, Hartley J, Saunders D, Montgomery M, Fiske J. Conceptos clave en comunicación y estudios culturales. Buenos Aires, Argentina: Amorrortu editores; 1997.

2. Daniels H. Vygostky y la pedagogía. Barcelona, España: Paidós; 2003.

3. Echeverría B. Definición de la Cultura. Ciudad de México, México: Fondo de Cultura Económica; 2019.

4. Peacock J. La lente antropológica. Madrid, España: Alianza Editorial; 2005.

5. French $\mathrm{M}$, Monahan $\mathrm{T}$. Dis-ease Surveillance: How Might Surveillance Studies Address COVID-19? Surveillance \& Society. 2020;18(1):1-11. Disponible en: https://bit.ly/3unTbuY

6. Giordano P. En tiempos de contagio. Barcelona, España: Narrativa Salamandra; 2020.

7. Žižek S. Pandemia. La covid-19 estremece al mundo. Barcelona, España: Anagrama; 2020.

8. Adreu-Periz D, Ochando-García A, LimónCáceres E. Experiencias de vida y soporte percibido por las enfermeras de las unidades de hemodiálisis durante la pandemia de COVID-19 en España. Enfermería 
Nefrológica. $\quad 2020 ; 23(2): 148-159$. https://doi.org/10.37551/S225428842020022

9. Lozano-Vargas A. Impacto de la epidemia del Coronavirus (COVID-19) en la salud mental del personal de salud y en la población general de China. Revista de Neuropsiquiatría. $\quad$ 2020;83(1): 51-56. http://dx.doi.org/10.20453/rnp.v83i1.3687

10. Zhou SJ, Zhang LG, Wang LL, et al. Prevalence and socio-demographic correlates of psychological health problems in Chinese adolescents during the outbreak of COVID-19. European child \& Adolescent Psychiatry. 2020;(29):749-758. https://doi.org/10.1007/s00787-02001541-4

11. Kim J. Learning and Teaching Online During Covid-19: Experiences of Student Teachers in an Early Childhood Education Practicum. International Journal of Early Childhood. 2020;(52):145-158. https://doi.org/10.1007/s13158-02000272-6

12. Wang C, Geale S. The power of story: narrative inquiry as a methodology in nursing research. International Journal of Nursing Sciences. 2015;2(2):195-198. https://doi.org/10.1016/j.ijnss.2015.04.014

13. Paulín G, Horta J, Siade, G. La vivencia y su análisis: consideraciones breves sobre las nociones objeto-sujeto en el universo discursivo del mundo cultural. Revista Mexicana de Ciencias Políticas y Sociales. 2009;(205):15-35. Disponible en: https://bit.ly/3ucInQy

14. Jameson F. Los estudios culturales. Buenos Aires, Argentina: Ediciones Godot; 2016.

15. Gibbs G. El análisis de datos cualitativos en Investigación Cualitativa. Madrid, España: Morata; 2012.

16. España S. La pandemia deja al $83 \%$ de los trabajadores en Ecuador en el desempleo o con condiciones precarias. El país. 26 de agosto de 2020.

17. LACC's Andean Studies Program (Productor). Impactos socioculturales del COVID-19 en Guayaquil, Ecuador: La intensificación de desigualdades. 2020. Disponible en: https://www.youtube.com/watch?v=DBSyf C9WR9o

\section{Conflicto de intereses}

La investigación se enmarcó en los estándares éticos, en pro de mejorar los procesos institucionales. No fue requerida ayuda financiera de ninguna institución gubernamental o no gubernamental.

Los autores declaran no tener ningún conflicto de intereses. 